\title{
Revisiting the Sequence Constraint
}

\author{
Willem-Jan van Hoeve ${ }^{1}$, Gilles Pesant ${ }^{2,3}$, \\ Louis-Martin Rousseau ${ }^{2,3,4}$, and Ashish Sabharwal ${ }^{1}$ \\ ${ }^{1}$ Department of Computer Science, Cornell University, \\ 4130 Upson Hall, Ithaca, NY 14853, USA \\ \{vanhoeve, sabhar\}@cs.cornell.edu \\ 2 École Polytechnique de Montréal, Montreal, Canada \\ ${ }^{3}$ Centre for Research on Transportation (CRT), \\ Université de Montréal, C.P. 6128, succ. Centre-ville, Montreal, H3C 3J7, Canada \\ ${ }^{4}$ Oméga Optimisation Inc. \\ \{pesant, louism\}@crt.umontreal.ca
}

\begin{abstract}
Many combinatorial problems, such as car sequencing and rostering, feature sequence constraints, restricting the number of occurrences of certain values in every subsequence of a given width. To date, none of the filtering algorithms proposed guaranteed domain consistency. In this paper, we present three filtering algorithms for the sequence constraint, with complementary strengths. One borrows ideas from dynamic programming; another reformulates it as a regular constraint; the last is customized. The last two algorithms establish domain consistency. Our customized algorithm does so in polynomial time, and can even be applied to a generalized sequence constraint for subsequences of variable widths. Experimental results show the practical usefulness of each.
\end{abstract}

\section{Introduction}

The sequence constraint was introduced by Beldiceanu and Contejean 4 as a set of overlapping among constraints. The constraint is also referred to as among_seq in [3]. An among constraint restricts the number of variables to be assigned to a value from a specific set. For example, consider a nurse-rostering problem in which each nurse can work at most 2 night shifts during every 7 consecutive days. The among constraint specifies the 2 -out-of-7 relation, while the sequence constraint imposes such among for every subsequence of 7 days.

Beldiceanu and Carlsson [2] have proposed a filtering algorithm for the sequence constraint, while Régin and Puget [10] have presented a filtering algorithm for the sequence constraint in combination with a global cardinality constraint 8 for a car sequencing application. Neither approach establishes domain consistency, however. As the constraint is inherent to many real-life problems, improved filtering could have a substantial industrial impact.

In this work we present three novel filtering algorithms for the sequence constraint. The first is based on dynamic programming concepts and runs in polynomial time, but it does not establish domain consistency. The second algorithm is based on the regular constraint [7]. It establishes domain consistency,

F. Benhamou (Ed.): CP 2006, LNCS 4204, pp. 620634 2006.

(C) Springer-Verlag Berlin Heidelberg 2006 
but needs exponential time in the worst case. In most practical cases it is very efficient however. Our third algorithm establishes domain consistency in polynomial time. It can be applied to a generalized version of the sequence constraint, for which the subsequences are of variable length. Moreover the number of occurrences may also vary per subsequence. Each algorithm has advantages over the others, either in terms of (asymptotic) running time or in terms of filtering.

The rest of the paper is structured as follows. Section 2 presents some background and notation on constraint programming. Section 3 recalls and discusses the among and sequence constraints. Sections 4 to 6 describe filtering algorithms for sequence. Section 7 compares the algorithms experimentally. Finally, Section 8 summarizes the contributions of the paper and discusses possible extensions.

\section{Constraint Programming Preliminaries}

We first introduce basic constraint programming concepts. For more information on constraint programming we refer to [1].

Let $x$ be a variable. The domain of $x$ is a set of values that can be assigned to $x$ and is denoted by $D(x)$. In this paper we only consider variables with finite domains. Let $X=x_{1}, x_{2}, \ldots, x_{k}$ be a sequence of variables. We denote $D(X)=$ $\bigcup_{1 \leq i \leq k} D\left(x_{i}\right)$. A constraint $C$ on $X$ is defined as a subset of the Cartesian product of the domains of the variables in $X$, i.e. $C \subseteq D\left(x_{1}\right) \times D\left(x_{2}\right) \times \cdots \times D\left(x_{k}\right)$. A tuple $\left(d_{1}, \ldots, d_{k}\right) \in C$ is called a solution to $C$. We also say that the tuple satisfies $C$. A value $d \in D\left(x_{i}\right)$ for some $i=1, \ldots, k$ is inconsistent with respect to $C$ if it does not belong to a tuple of $C$, otherwise it is consistent. $C$ is inconsistent if it does not contain a solution. Otherwise, $C$ is called consistent.

A constraint satisfaction problem, or a CSP, is defined by a finite sequence of variables $\mathcal{X}=x_{1}, x_{2}, \ldots, x_{n}$, together with a finite set of constraints $\mathcal{C}$, each on a subsequence of $\mathcal{X}$. The goal is to find an assignment $x_{i}=d_{i}$ with $d_{i} \in D\left(x_{i}\right)$ for $i=1, \ldots, n$, such that all constraints are satisfied. This assignment is called a solution to the CSP.

The solution process of constraint programming interleaves constraint propagation, or propagation in short, and search. The search process essentially consists of enumerating all possible variable-value combinations, until we find a solution or prove that none exists. We say that this process constructs a search tree. To reduce the exponential number of combinations, constraint propagation is applied to each node of the search tree: Given the current domains and a constraint $C$, remove domain values that do not belong to a solution to $C$. This is repeated for all constraints until no more domain value can be removed. The removal of inconsistent domain values is called filtering.

In order to be effective, filtering algorithms should be efficient, because they are applied many times during the solution process. Further, they should remove as many inconsistent values as possible. If a filtering algorithm for a constraint $C$ removes all inconsistent values from the domains with respect to $C$, we say that it makes $C$ domain consistent. Formally: 
Definition 1 (Domain consistency, [6]). A constraint $C$ on the variables $x_{1}, \ldots, x_{k}$ is called domain consistent if for each variable $x_{i}$ and each value $d_{i} \in D\left(x_{i}\right)(i=1, \ldots, k)$, there exist a value $d_{j} \in D\left(x_{j}\right)$ for all $j \neq i$ such that $\left(d_{1}, \ldots, d_{k}\right) \in C$.

In the literature, domain consistency is also referred to as hyper-arc consistency or generalized-arc consistency.

Establishing domain consistency for binary constraints (constraints defined on two variables) is inexpensive. For higher arity constraints this is not necessarily the case since the naive approach requires time that is exponential in the number of variables. Nevertheless the underlying structure of a constraint can sometimes be exploited to establish domain consistency much more efficiently.

\section{The Among and Sequence Constraints}

The among constraint restricts the number of variables to be assigned to a value from a specific set:

Definition 2 (Among constraint, [4]). Let $X=x_{1}, x_{2}, \ldots, x_{q}$ be a sequence of variables and let $S$ be a set of domain values. Let $0 \leq \min \leq \max \leq q$ be constants. Then

$$
\begin{aligned}
\operatorname{among}(X, S, \min , \max )= & \left\{\left(d_{1}, \ldots, d_{q}\right) \mid \forall i \in\{1, \ldots, q\} d_{i} \in D\left(x_{i}\right),\right. \\
& \left.\min \leq\left|\left\{i \in\{1, \ldots, q\} \quad: d_{i} \in S\right\}\right| \leq \max \right\} .
\end{aligned}
$$

Establishing domain consistency for the among constraint is not difficult. Subtracting from min, $\max$, and $q$ the number of variables that must take their value in $S$, and subtracting further from $q$ the number of variables that cannot take their value in $S$, we are in one of four cases:

1. $\max <0$ or $\min >q$ : the constraint is inconsistent;

2. $\max =0$ : remove values in $S$ from the domain of all remaining variables, making the constraint domain consistent;

3. $\min =q$ : remove values not in $S$ from the domain of all remaining variables, making the constraint domain consistent;

4. $\max >0$ and $\min <q$ : the constraint is already domain consistent.

The sequence constraint applies the same among constraint on every $q$ consecutive variables:

Definition 3 (Sequence constraint, [4]). Let $X=x_{1}, x_{2}, \ldots, x_{n}$ be an ordered sequence of variables (according to their respective indices) and let $S$ be a set of domain values. Let $1 \leq q \leq n$ and $0 \leq \min \leq \max \leq q$ be constants. Then

$$
\text { sequence }(X, S, q, \min , \max )=\bigwedge_{i=1}^{n-q+1} \operatorname{among}\left(s_{i}, S, \min , \max \right),
$$

where $s_{i}$ represents the sequence $x_{i}, \ldots, x_{i+q-1}$. 
In other words, the sequence constraint states that every sequence of $q$ consecutive variables is assigned to at least min and at most max values in $S$. Note that working on each among constraint separately, and hence locally, is not as powerful as reasoning globally. In particular, establishing domain consistency on each among of the conjunction does not ensure domain consistency for sequence.

Example 1. Let $X=x_{1}, x_{2}, x_{3}, x_{4}, x_{5}, x_{6}, x_{7}$ be an ordered sequence of variables variables with domains $D\left(x_{i}\right)=\{0,1\}$ for $i \in\{3,4,5,7\}, D\left(x_{1}\right)=D\left(x_{2}\right)=\{1\}$, and $D\left(x_{6}\right)=\{0\}$. Consider the constraint sequence $(X,\{1\}, 5,2,3)$, i.e., every sequence of five consecutive variables must account for two or three 1's. Each individual among is domain consistent but it is not the case for sequence: value 0 is unsupported for variable $x_{7}$. ( $x_{7}=0$ forces at least two 1 's among $\left\{x_{3}, x_{4}, x_{5}\right\}$, which brings the number of 1's for the leftmost among to at least four.)

Establishing domain consistency for the sequence constraint is not nearly as easy as for among. The algorithms proposed so far in the literature may miss such global reasoning. The filtering algorithm proposed in 10 and implemented in Ilog Solver does not filter out 0 from $D\left(x_{7}\right)$ in the previous example. However in some special cases domain consistency can be efficiently computed: When min equals max, it can be established in linear time. Namely, if there is a solution, then $x_{i}$ must equal $x_{i+q}$ because of the constraints $a_{i}+a_{i+1}+\cdots+a_{i+q-1}=\min$ and $a_{i+1}+\cdots+a_{i+q}=\min$. Hence, if one divides the sequence up into $n / q$ consecutive subsequences of size $q$ each, they must all look exactly the same. Thus, establishing domain consistency now amounts to propagating the "settled" variables (i.e. $D\left(x_{i}\right) \subseteq S$ or $D\left(x_{i}\right) \cap S=\varnothing$ ) to the first subsequence and then applying the previously described algorithm for among. Two of the filtering algorithms we describe below establish domain consistency in the general case.

Without loss of generality, we shall consider instances of sequence in which $S=\{1\}$ and the domain of each variable is a subset of $\{0,1\}$. Using an element constraint, we can map every value in $S$ to 1 and every other value (i.e., $D(X) \backslash S$ ) to 0 , yielding an equivalent instance on new variables.

\section{A Graph-Based Filtering Algorithm}

We propose a first filtering algorithm that considers the individual among constraints of which the sequence constraint is composed. First, it filters the among constraints for each sequence of $q$ consecutive variables $s_{i}$. Then it filters the conjunction of every pair of consecutive sequences $s_{i}$ and $s_{i+1}$. This is presented as SuccessiveLocalGraph (SLG) in Algorithm 1, and discussed below.

\subsection{Filtering the among Constraints}

The individual among constraints are filtered with the algorithm FilterLocALGraPH. For each sequence $s_{i}=x_{i}, \ldots, x_{i+q-1}$ of $q$ consecutive variables in 

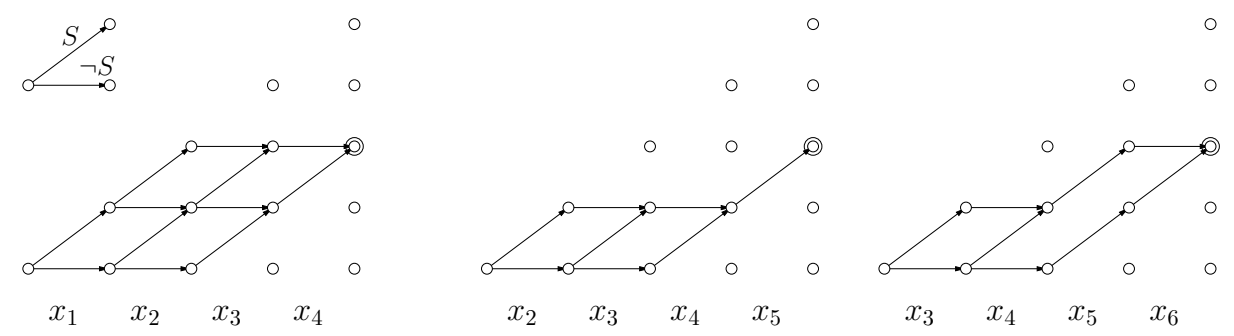

Fig. 1. Filtered Local Graphs of Example 2

$X=x_{1}, \ldots, x_{n}$, we build a digraph $G_{s_{i}}=\left(V_{i}, A_{i}\right)$ as follows. The vertex set and the arc set are defined as

$$
\begin{gathered}
V_{i}=\left\{v_{j, k} \mid j \in\{i-1, \ldots, i+q-1\}, k \in\{0, \ldots, j\}\right\}, \\
A_{i}=\left\{\left(v_{j, k}, v_{j+1, k}\right) \mid j \in\{i-1, \ldots, i+q-2\}, k \in\{0, \ldots, j\}, D\left(x_{j+1}\right) \backslash S \neq \emptyset\right\} \cup \\
\left\{\left(v_{j, k}, v_{j+1, k+1}\right) \mid j \in\{i-1, \ldots, i+q-2\}, k \in\{0, \ldots, j\}, D\left(x_{j+1}\right) \cap S \neq \emptyset\right\} .
\end{gathered}
$$

In other words, the arc $\left(v_{j, k}, v_{j+1, k+1}\right)$ represents variable $x_{j+1}$ taking its value in $S$, while the arc $\left(v_{j, k}, v_{j+1, k}\right)$ represents variable $x_{j+1}$ not taking its value in $S$. The index $k$ in $v_{j, k}$ represents the number of variables in $x_{i}, \ldots, x_{j-1}$ that take their value in $S$. This is similar to the dynamic programming approach taken in 11 to filter knapsack constraints.

Next, the individual among constraint on sequence $s_{i}$ is filtered by removing all arcs that are not on a path from vertex $v_{i-1,0}$ to a goal vertex $v_{i+q-1, k}$ with $\min \leq k \leq \max$. This can be done in linear time (in the size of the graph, $\left.\Theta\left(q^{2}\right)\right)$ by breadth-first search starting from the goal vertices. Naturally, if the filtered graph contains no arc $\left(v_{j, k}, v_{j+1, k}\right)$ for all $k$, we remove $S$ from $D\left(x_{j+1}\right)$. Similarly, we remove $D(X) \backslash S$ from $D\left(x_{j+1}\right)$ if it contains no $\operatorname{arc}\left(v_{j, k}, v_{j+1, k+1}\right)$ for all $k$.

Example 2. Let $X=x_{1}, x_{2}, x_{3}, x_{4}, x_{5}, x_{6}$ be an ordered sequence of variables with domains $D\left(x_{i}\right)=\{0,1\}$ for $i \in\{1,2,3,4,6\}$ and $D\left(x_{5}\right)=\{1\}$. Let $S=\{1\}$. Consider the constraint sequence $(X, S, 4,2,2)$. The filtered local graphs of this constraint are depicted in Figure 1 .

\subsection{Filtering for a Sequence of among}

We filter the conjunction of two "consecutive" among constraints. This algorithm has a "forward" phase and a "backward" phase. In the forward phase, we compare the among on $s_{i}$ with the among on $s_{i+1}$ for increasing $i$, using the algorithm COMPARE. This is done by projecting $G_{s_{i+1}}$ onto $G_{s_{i}}$ such that corresponding variables overlap. Doing so, the projection keeps only arcs that appear in both original local graphs. We can either project vertex $v_{i+1,0}$ of $G_{s_{i+1}}$ onto vertex $v_{i+1,0}$ of $G_{s_{i}}$, or onto vertex $v_{i+1,1}$ of $G_{s_{i}}$. We consider both projections separately, and label all arcs "valid" if they belong to a path from vertex $v_{i, 0}$ to 
Algorithm 1. Filtering algorithm for the sequence constraint

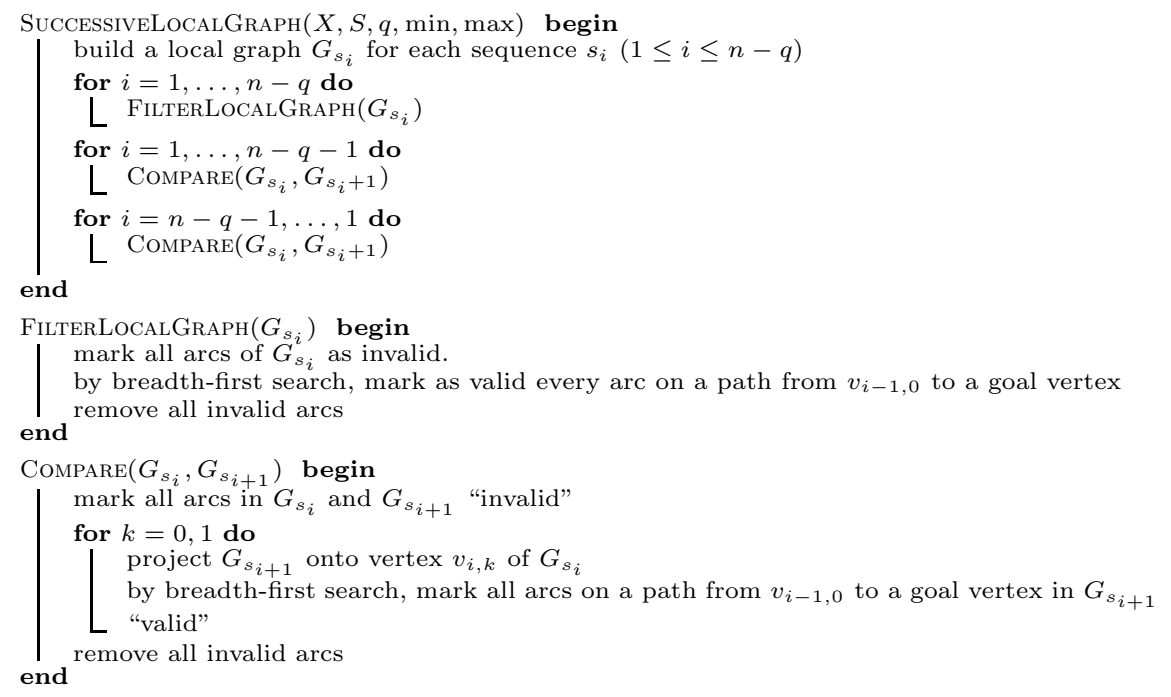

goal vertex in $G_{s_{i+1}}$ in one of the composite graphs. All other arcs are labeled "invalid", and are removed from the original graphs $G_{s_{i}}$ and $G_{s_{i+1}}$. In the backward phase, we compare the among on $s_{i}$ with the among on $s_{i+1}$ for decreasing $i$, similarly to the forward phase.

\subsection{Analysis}

SuCCESSIVELocalGraph does not establish domain consistency for the sequence constraint. We illustrate this in the following example.

Example 3. Let $X=x_{1}, x_{2}, \ldots, x_{10}$ be an ordered sequence of variables with domains $D\left(x_{i}\right)=\{0,1\}$ for $i \in\{3,4,5,6,7,8\}$ and $D\left(x_{i}\right)=\{0\}$ for $i \in\{1,2,9,10\}$. Let $S=\{1\}$. Consider the constraint sequence $(X, S, 5,2,3)$, i.e., every sequence of 5 consecutive variables must take between 2 and 3 values in $S$. The first among constraint imposes that at least two variables out of $\left\{x_{3}, x_{4}, x_{5}\right\}$ must be 1. Hence, at most one variable out of $\left\{x_{6}, x_{7}\right\}$ can be 1 , by the third among. This implies that $x_{8}$ must be 1 (from the last among). Similarly, we can deduce that $x_{3}$ must be 1 . This is however not deduced by our algorithm.

The problem occurs in the COMPARE method, when we merge the valid arcs coming from different projection. Up until that point there is a direct equivalence between a path in a local graph and a support for the constraint. However the union of the two projection breaks this equivalence and thus prevents this algorithm from being domain consistent.

The complexity of the algorithm is polynomial since the local graphs are all of size $O(q \cdot \max )$. Hence FilterLocalGraph runs in $O(q \cdot \max )$ time, which is called $n-q$ times. The algorithm COMPARE similarly runs for $O(q \cdot \max )$ steps 
and is called $2(n-q)$ times. Thus, the filtering algorithm runs in $O((n-q) \cdot q \cdot \max )$ time. As $\max \leq q$, it follows that the algorithm runs in $O\left(n q^{2}\right)$ time.

\section{Reaching Domain Consistency Through regular}

The regular constraint [7, defining the set of allowed tuples for a sequence of variables as the language recognized by a given automaton, admits an incremental filtering algorithm establishing domain consistency. In this section, we give an automaton recognizing the tuples of the sequence constraint whose number of states is potentially exponential in $q$. Through that automaton, we can express sequence as a regular constraint, thereby obtaining domain consistency.

The idea is to record in a state the last $q$ values encountered, keeping only the states representing valid numbers of 1 's for a sequence of $q$ consecutive variables and adding the appropriate transitions between those states. Let $Q_{k}^{q}$ denote the set of strings of length $q$ featuring exactly $k 1$ 's and $q-k 0$ 's - there are $\left(\begin{array}{l}q \\ k\end{array}\right)$ such strings. Given the constraint sequence $(X,\{1\}, q, \ell, u)$, we create states for each of the strings in $\bigcup_{k=\ell}^{u} Q_{k}^{q}$. By a slight abuse of notation, we will refer to a state using the string it represents. Consider a state $d_{1} d_{2} \ldots d_{q}$ in $Q_{k}^{q}, \ell \leq k \leq u$. We add a transition on 0 to state $d_{2} d_{3} \ldots d_{q} 0$ if and only if $d_{1}=0 \vee\left(d_{1}=1 \wedge k>\ell\right)$. We add a transition on 1 to state $d_{2} d_{3} \ldots d_{q} 1$ if and only if $d_{1}=1 \vee\left(d_{1}=0 \wedge k<u\right)$.

We must add some other states to encode the first $q-1$ values of the sequence: one for the initial state, two to account for the possible first value, four for the first two values, and so forth. There are at most $2^{q}-1$ of those states, considering that some should be excluded because the number of 1's does not fall within $[\ell, u]$. More precisely, we will have states

$$
\bigcup_{i=0}^{q-1} \bigcup_{k=\max (0, \ell-(q-i))}^{\min (i, u)} Q_{k}^{i}
$$

Transitions from a state $d_{1} \ldots d_{i}$ in $Q_{k}^{i}$ to state $d_{1} \ldots d_{i} 0$ in $Q_{k}^{i+1}$ on value 0 and to state $d_{1} \ldots d_{i} 1$ in $Q_{k+1}^{i+1}$ on value 1 , provided such states are part of the automaton. Every state in the automaton is considered a final (accepting) state. Figure 2 illustrates the automaton that would be built for the constraint sequence $(X,\{1\}, 4,1,2)$.

The filtering algorithm for regular guarantees domain consistency provided that the automaton recognizes precisely the solutions of the constraint. By construction, the states $Q_{\star}^{q}$ of the automaton represent all the valid configurations of $q$ consecutive values and the transitions between them imitate a shift to the right over the sequence of values. In addition, the states $Q_{\star}^{i}, 0 \leq i<q$ are linked so that the first $q$ values reach a state that encodes them. All states are accepting states so the sequence of $n$ values is accepted if and only if the automaton completes the processing. Such a completion corresponds to a successful scan of every subsequence of length $q$, precisely our solutions. 

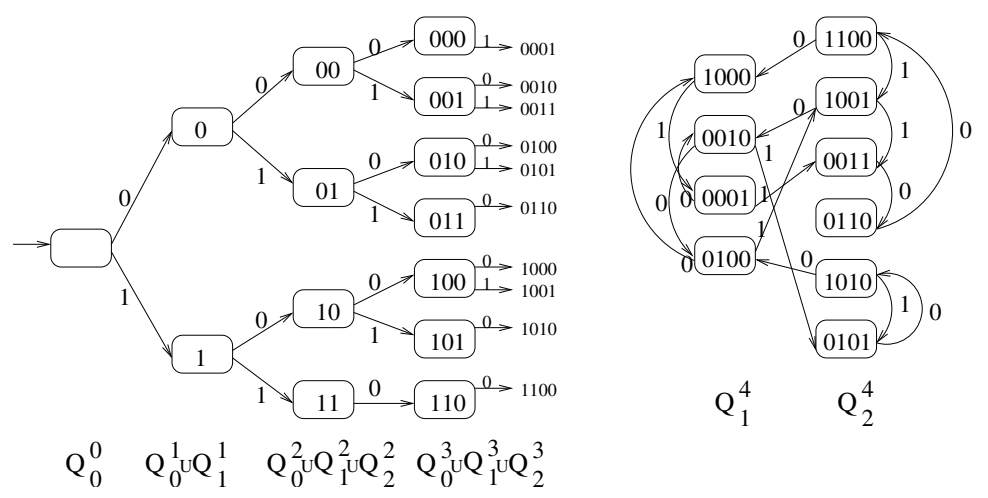

Fig. 2. Automaton for sequence $(X,\{1\}, 4,1,2)$

The resulting algorithm runs in time linear in the size of the underlying graph, which has $O\left(n 2^{q}\right)$ vertices and arcs in the worst case. Nevertheless, in most practical problems $q$ is much smaller than $n$. Note also that subsequent calls of the algorithm run in time proportional to the number of updates in the graph and not to the size of the whole graph.

\section{Reaching Domain Consistency in Polynomial Time}

The filtering algorithms we considered thus far apply to sequence constraints with fixed among constraints for the same $q$, min, and max. In this section we present a polynomial-time algorithm that achieves domain consistency in a more generalized setting, where we have $m$ arbitrary among constraints over sequences of consecutive variables in $X$. These $m$ constraints may have different min and max values, be of different length, and overlap in an arbitrary fashion. A conjunction of $k$ sequence constraints over the same ordered set of variables, for instance, can be expressed as a single generalized sequence constraint. We define the generalized sequence constraint, gen-sequence, formally as follows:

Definition 4 (Generalized sequence constraint). Let $X=x_{1}, \ldots, x_{n}$ be an ordered sequence of variables (according to their respective indices) and $S$ be a set of domain values. For $1 \leq j \leq m$, let $s_{j}$ be a sequence of consecutive variables in $X,\left|s_{j}\right|$ denote the length of $s_{j}$, and integers $\min _{j}$ and $\max _{j}$ be such that $0 \leq \min _{j} \leq \max _{j} \leq\left|s_{j}\right|$. Let $\Sigma=\left\{s_{1}, \ldots, s_{m}\right\}, \operatorname{Min}=\left\{\min _{1}, \ldots, \min _{m}\right\}$, and $\operatorname{Max}=\left\{\max _{1}, \ldots, \max _{m}\right\}$. Then

$$
\text { gen-sequence }(X, S, \Sigma, \operatorname{Min}, \operatorname{Max})=\bigwedge_{j=1}^{m} \operatorname{among}\left(s_{j}, S, \min _{j}, \max _{j}\right) \text {. }
$$

For simplicity, we will identify each $s_{j} \in \Sigma$ with the corresponding among constraint on $s_{j}$. The basic structure of the filtering algorithm for the gen-sequence 
Algorithm 2. Complete filtering algorithm for the gen-sequence constraint

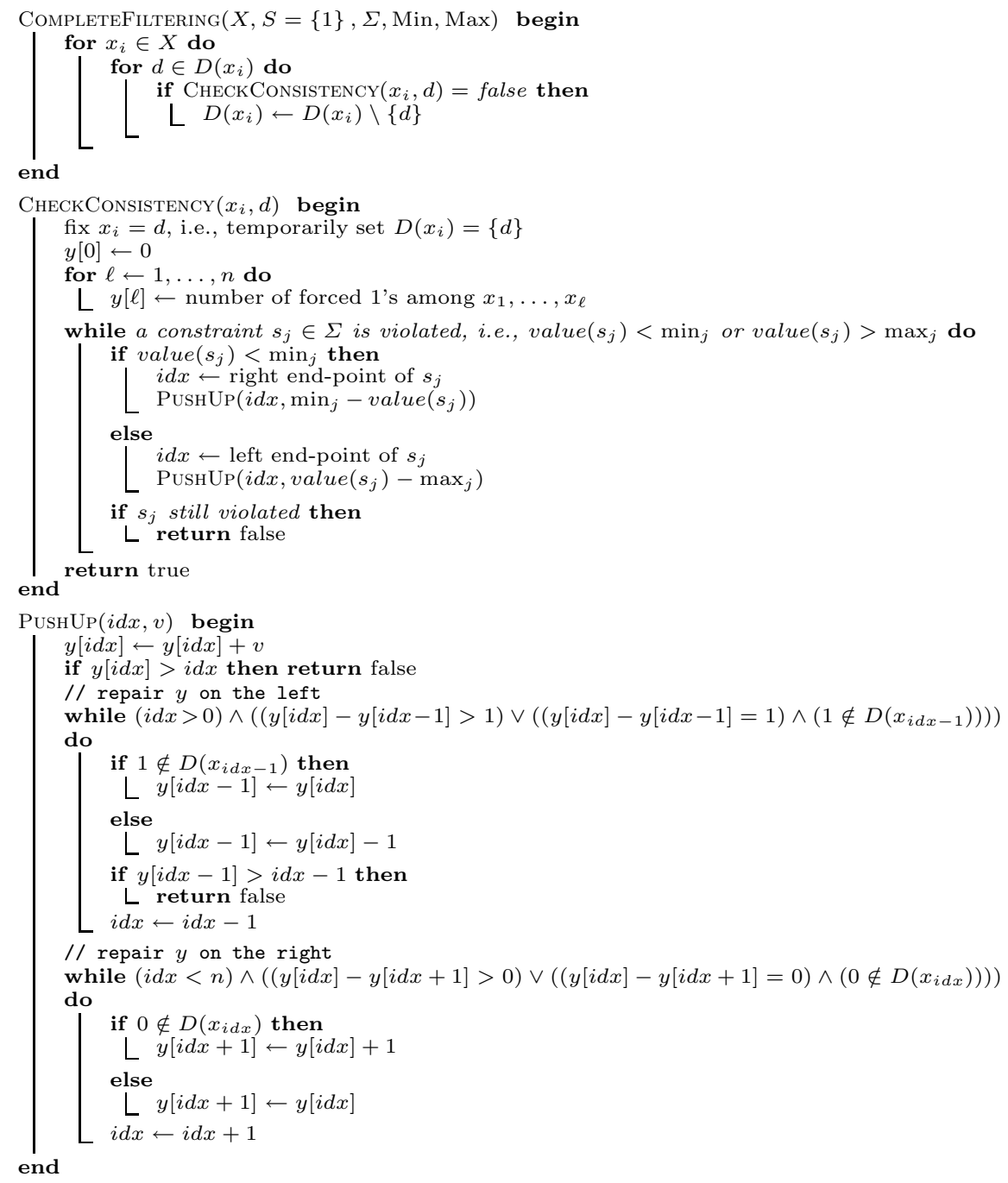

constraint is presented as Algorithm 2. The main loop, CompleteFiltering, simply considers all possible domain values of all variables. If a domain value is yet unsupported, we check its consistency via procedure CHECKCONSISTENCY. If it has no support, we remove it from the domain of the corresponding variable.

Procedure CHECKCONSISTENCY is the heart of the algorithm. It finds a single solution to the gen-sequence constraint, or proves that none exists. It uses a single array $y$ of length $n+1$, such that $y[0]=0$ and $y[i]$ represents the number of 1 's among $x_{1}, \ldots, x_{i}$. The invariant for $y$ maintained throughout is that $y[i+1]-y[i]$ is either 0 or 1 . Initially, we start with the lowest possible array, in which $y$ is filled according to the lower bounds of the variables in $X$. 
For clarity, let $L_{j}$ and $R_{j}$ denote the left and right end-points, respectively, of the among constraint $s_{j} \in \Sigma ; R_{j}=L_{j}+\left|s_{j}\right|-1$. As an example, for the usual sequence constraint with among constraints of size $q, L_{j}$ would be $i$ and $R_{j}$ would be $i+q-1$. The value of $s_{j}$ is computed using the array $y$ : value $\left(s_{j}\right)=$ $y\left[R_{j}\right]-y\left[L_{j}-1\right]$. In other words, value $\left(s_{j}\right)$ counts exactly the number of 1 's in the sequence $s_{j}$. Hence, a constraint $s_{j}$ is satisfied if and only if $\min _{j} \leq$ value $\left(s_{j}\right) \leq \max _{j}$. In order to find a solution, we consider all among constraints $s_{j} \in \Sigma$. Whenever a constraint $s_{j}$ is violated, we make it consistent by "pushing up" either $y\left[R_{j}\right]$ or $y\left[L_{j}-1\right]$ :

if value $\left(s_{j}\right)<\min _{j}$, then push up $y\left[R_{j}\right]$ with value $\min _{j}-\operatorname{value}\left(s_{j}\right)$, if value $\left(s_{j}\right)>\max _{j}$, then push up $y\left[L_{j}-1\right]$ with value value $\left(s_{j}\right)-\max _{j}$.

Such a "push up" may result in the invariant for $y$ being violated. We therefore repair $y$ in a minimal fashion to restore its invariant as follows. Let $y[i d x]$ be the entry that has been pushed up. We first push up its neighbors on the left side (from $i d x$ downward). In case $x_{i d x-1}$ is fixed to 0 , we push up $y[i d x-1]$ to the same level $y[i d x]$. Otherwise, we push it up to $y[i d x]-1$. This continues until the difference between all neighbors is at most 1 . Whenever $y[i]>i$ for some $i$, we need more 1's than there are variables up to $i$, and we report an immediate failure. Repairing the array on the right side is done in a similar way.

Example 4. Consider again the sequence constraint from Example 2, i.e., the constraint sequence $(X, S, 4,2,2)$ with $X=\left\{x_{1}, x_{2}, x_{3}, x_{4}, x_{5}, x_{6}\right\}, D\left(x_{i}\right)=$ $\{0,1\}$ for $i \in\{1,2,3,4,6\}, D\left(x_{5}\right)=\{1\}$, and $S=\{1\}$. The four among constraints are over $s_{1}=\left\{x_{1}, x_{2}, x_{3}\right\}, s_{2}=\left\{x_{2}, x_{3}, x_{4}\right\}, s_{3}=\left\{x_{3}, x_{4}, x_{5}\right\}$, and $s_{4}=\left\{x_{4}, x_{5}, x_{6}\right\}$. We apply CHECKCONSISTENCY to find a minimum solution. The different steps are depicted in Figure 3 We start with $y=[0,0,0,0,0,1,1]$, and consider the different among constraints. First we consider $s_{1}$, which is violated. Namely, value $\left(s_{1}\right)=y[3]-y[0]=0-0=0$, while it should be at least 2 . Hence, we push up $y[3]$ with 2 units, and obtain $y=[0,0,1,2,2,3,3]$. Note that we push up $y[5]$ to 3 because $x_{5}$ is fixed to 1 .

Next we consider $s_{2}$ with value $y[4]-y[1]=2$, which is not violated. We continue with $s_{3}$ with value $y[5]-y[2]=2$, which is not violated. Then we consider $s_{4}$ with value $y[6]-y[3]=1$, which is violated as it should be at least 2 . Hence, we push up $y[6]$ by 1 , and obtain $y=[0,0,1,2,2,3,4]$. One more loop over the among constraint concludes consistency, with minimum solution $x_{1}=0, x_{2}=1, x_{3}=1, x_{4}=0, x_{5}=1, x_{6}=1$.

We have optimized the basic procedure in Algorithm2 in several ways. The main loop of COMPLETEFILTERING is improved by maintaining a support for all domain values. Namely, one call to CHECKCONSISTENCY (with positive response) yields a support for $n$ domain values. This immediately reduces the number of calls to CHeckConsistency by half, while in practice the reduction is even more. A second improvement is achieved by starting out CompleteFilterING with the computation of the "minimum" and the "maximum" solutions to gen-sequence, in a manner very similar to the computation in CHECKConsisTENCY but without restricting the value of any variable. This defines bounds 

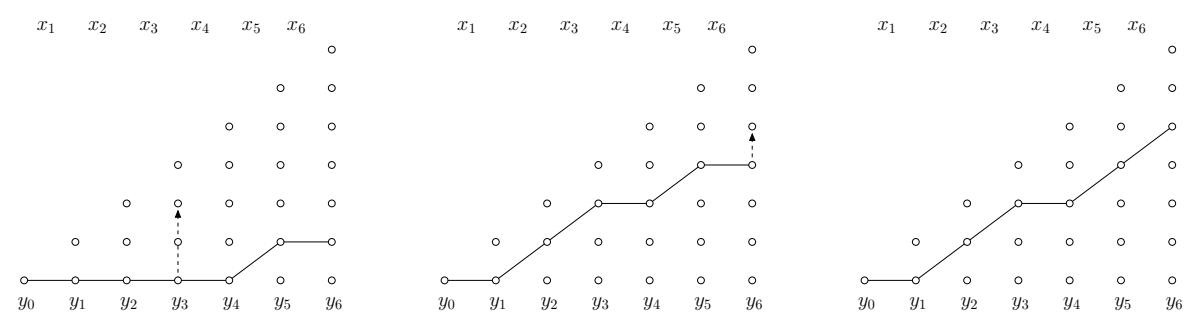

Fig. 3. Finding a minimum solution to Example 4

$y_{\min }$ and $y_{\max }$ within which $y$ must lie for all subsequent consistency checks (details in the following section).

\subsection{Analysis}

A solution to a gen-sequence constraint can be thought of as the corresponding binary sequence or, equivalently, as the $y$ array for it. This $y$ array representation has a useful property. Let $y$ and $y^{\prime}$ be two solutions. Define array $y \oplus y^{\prime}$ to be the smaller of $y$ and $y^{\prime}$ at each point, i.e., $\left(y \oplus y^{\prime}\right)[i]=\min \left(y[i], y^{\prime}[i]\right)$.

Lemma 1. If $y, y^{\prime}$ are solutions to a gen-sequence constraint, then so is $y \oplus y^{\prime}$.

Proof. Suppose for the sake of contradiction that $y^{*}=y \oplus y^{\prime}$ violates an among constraint $s$ of the gen-sequence constraint. Let $L$ and $R$ denote the left and right end-points of $s$, respectively. Suppose $y^{*}$ violates the min constraint, i.e., $y^{*}[R]-y^{*}[L-1]<\min (s)$. Since $y$ and $y^{\prime}$ satisfy $s$, it must be that $y^{*}$ agrees with $y$ on one end-point of $s$ and with $y^{\prime}$ on the other. W.l.o.g., assume $y^{*}[L-1]=y^{\prime}[L-1]$ and $y^{*}[R]=y[R]$. By the definition of $y^{*}$, it must be that $y[L-1] \geq y^{\prime}[L-1]$, so that $y[R]-y[L-1] \leq y[R]-y^{\prime}[L-1]=y^{*}[R]-y^{*}[L-1]<$ $\min (s)$. In other words, $y$ itself violates $s$, a contradiction. A similar reasoning works when $y^{*}$ violates the max constraint of $s$.

As a consequence of this property, we can unambiguously define an absolute minimum solution for gen-sequence as the one whose $y$ value is the lowest over all solutions. Denote this solution by $y_{\min }$; we have that for all solutions $y$ and for all $i, y_{\min }[i] \leq y[i]$. Similarly, define the absolute maximum solution, $y_{\max }$.

Lemma 2. The procedure CHECKCONSISTENCY constructs the minimum solution to the gen-sequence constraint or proves that none exists, in $O\left(n^{2}\right)$ time.

Proof. CheckConsistency reports success only when no among constraint in gen-sequence is violated by the current $y$ values maintained by it, i.e., $y$ is a solution. Hence, if there is no solution, this fact is detected. We will argue that when CHeckConsistency does report success, its $y$ array exactly equals $y_{\text {min }}$.

We first show by induction that $y$ never goes above $y_{\min }$ at any point, i.e., $y[i] \leq y_{\min }[i], 0 \leq i \leq n$ throughout the procedure. For the base case, $y[i]$ is 
clearly initialized to a value not exceeding $y_{\min }[i]$, and the claim holds trivially. Assume inductively that the claim holds after processing $t \geq 0$ among constraint violations. Let $s$ be the $t+1^{s t}$ violated constraint processed. We will show that the claim still holds after processing $s$.

Let $L$ and $R$ denote the left and right end-points of $s$, respectively. First consider the case that the min constraint was violated, i.e., $y[R]-y[L-1]<$ $\min (s)$, and index $L-1$ was pushed up so that the new value of $y[L-1]$, denoted $\hat{y}[L-1]$, became $y[R]-\min (s)$. Since this was the first time a $y$ value exceeded $y_{\text {min }}$, we have $y[R] \leq y_{\min }[R]$, so that $\hat{y}[L-1] \leq y_{\min }[R]-\min (s) \leq y_{\min }[L-1]$. It follows that $\hat{y}[L-1]$ itself does not exceed $y_{\min }[L-1]$. It may still be that the resulting repair on the left or the right causes a $y_{\min }$ violation. However, the repair operations only lift up $y$ values barely enough to be consistent with the possible domain values of the relevant variables. In particular, repair on the right "flattens out" $y$ values to equal $\hat{y}[L-1]$ (forced 1's being exceptions) as far as necessary to "hit" the solution again. It follows that since $\hat{y}[L-1] \leq y_{\min }[L-1]$, all repaired $y$ values must also not go above $y_{\min }$. A similar argument works when instead the max constraint is violated. This finishes the inductive step.

This shows that by performing repeated PushUp operations, one can never accidentally "go past" the solution $y_{\text {min }}$. Further, since each PusHUP increases $y$ in at least one place, repeated calls to it will eventually "hit" $y_{\min }$ as a solution.

For the time complexity of CheckConsistency, note that $y[i] \leq i$. Since we monotonically increase $y$ values, we can do so at most $\sum_{i=1}^{n} i=O\left(n^{2}\right)$ times. The cost of each PushUp operation can be charged to the $y$ values it changes because the while loops in it terminate as soon as they find a $y$ value that need not be changed. Finally, simple book-keeping can be used to locate a violated constraint in constant time. This proves the desired bound of $O\left(n^{2}\right)$ overall.

The simple loop structure of COMPLETEFILTERING immediately implies:

Theorem 1. Algorithm CompleteFiltering establishes domain consistency on the gen-sequence constraint or proves that it is inconsistent, in $O\left(n^{3}\right)$ time.

Remark 1. Régin proved that finding a solution to an arbitrary combination of among constraints is NP-complete [9. Our algorithm finds a solution in polynomial time to a more restricted problem, namely, when each among constraint is defined on a sequence of consecutive variables with respect to a fixed ordering.

\section{$7 \quad$ Experimental Results}

To evaluate the different filtering algorithms presented, we used two sets of benchmark problems. The first is a very simple model, constructed with only one sequence constraint, allowing us to isolate and evaluate the performance of each method. Then we conduct a limited series of experiments on the well-known car sequencing problem. Successive Local Graph (SLG), Generalized Sequence (GS), and regular-based implementation (REG) are compared with the sequence constraint provided in the Ilog Solver library in both basic (IB) and 
Table 1. Comparison on instances with $n=100, d=10$

\begin{tabular}{|rr|rr|rr|rr|rr|rr|}
\hline & & \multicolumn{2}{|c|}{ IB } & \multicolumn{2}{|c|}{ IE } & \multicolumn{2}{|c|}{ SLG } & \multicolumn{2}{|c|}{ GS } & \multicolumn{2}{|c|}{ REG } \\
$q$ & $\Delta$ & $\mathrm{BT}$ & $\mathrm{CPU}$ & $\mathrm{BT}$ & $\mathrm{CPU}$ & $\mathrm{BT}$ & $\mathrm{CPU}$ & $\mathrm{BT}$ & $\mathrm{CPU}$ & $\mathrm{BT}$ & $\mathrm{CPU}$ \\
\hline 5 & 1 & - & - & 33976.9 & 18.210 & 0.2 & 0.069 & 0 & 0.014 & 0 & 0.009 \\
6 & 2 & 361770 & 54.004 & 19058.3 & 6.390 & 0 & 0.078 & 0 & 0.013 & 0 & 0.018 \\
7 & 1 & 380775 & 54.702 & 113166 & 48.052 & 0 & 0.101 & 0 & 0.012 & 0 & 0.020 \\
7 & 2 & 264905 & 54.423 & 7031 & 4.097 & 0 & 0.129 & 0 & 0.016 & 0 & 0.039 \\
7 & 3 & 286602 & 48.012 & 0 & 0.543 & 0 & 0.129 & 0 & 0.015 & 0 & 0.033 \\
9 & 1 & - & - & 60780.5 & 42.128 & 0.1 & 0.163 & 0 & 0.010 & 0 & 0.059 \\
9 & 3 & 195391 & 43.024 & 0 & 0.652 & 0 & 0.225 & 0 & 0.016 & 0 & 0.187 \\
\hline
\end{tabular}

Table 2. Comparison on instances with $\Delta=1, d=10$

\begin{tabular}{|rr|rr|rr|rr|rr|rr|}
\hline & & \multicolumn{2}{|c|}{ IB } & \multicolumn{2}{|c|}{ IE } & \multicolumn{2}{|c|}{ SLG } & \multicolumn{2}{|c|}{ GS } & \multicolumn{2}{c|}{ REG } \\
$q$ & $n$ & BT & CPU & BT & CPU & BT & CPU & BT & CPU & BT & CPU \\
\hline 5 & 50 & 459154 & 18.002 & 22812 & 18.019 & 0.4 & 0.007 & 0 & 0.001 & 0 & 0.001 \\
5 & 100 & 192437 & 12.008 & 11823 & 12.189 & 1 & 0.041 & 0 & 0.005 & 0 & 0.005 \\
5 & 500 & 48480 & 12.249 & 793 & 41.578 & 0.7 & 1.105 & 0 & 0.466 & 0 & 0.023 \\
5 & 1000 & 942 & 1.111 & 2.3 & 160.000 & 1.1 & 5.736 & 0 & 4.374 & 0 & 0.062 \\
\hline 7 & 50 & 210107 & 12.021 & 67723 & 12.309 & 0.2 & 0.015 & 0 & 0.001 & 0 & 0.006 \\
7 & 100 & 221378 & 18.030 & 44963 & 19.093 & 0.4 & 0.059 & 0 & 0.005 & 0 & 0.010 \\
7 & 500 & 80179 & 21.134 & 624 & 48.643 & 2.8 & 2.115 & 0 & 0.499 & 0 & 0.082 \\
7 & 1000 & 30428 & 28.270 & 46 & 138.662 & 588.5 & 14.336 & 0 & 3.323 & 0 & 0.167 \\
\hline 9 & 50 & 18113 & 1.145 & 18113 & 8.214 & 0.9 & 0.032 & 0 & 0.001 & 0 & 0.035 \\
9 & 100 & 3167 & 0.306 & 2040 & 10.952 & 1.6 & 0.174 & 0 & 0.007 & 0 & 0.087 \\
9 & 500 & 48943 & 18.447 & 863 & 65.769 & 2.2 & 4.311 & 0 & 0.485 & 0 & 0.500 \\
9 & 1000 & 16579 & 19.819 & 19 & 168.624 & 21.9 & 16.425 & 0 & 3.344 & 0 & 0.843 \\
\hline
\end{tabular}

extended (IE) propagation modes. Experiments were run with Ilog Solver 6.2 on a bi-processor Intel Xeon HT 2.8Ghz, 3G RAM.

\subsection{Single Sequence}

To evaluate the filtering both in terms of domain reduction and efficiency, we build a very simple model consisting of only one sequence constraint.

The first series of instances is generated in the following manner. All instances contain $n$ variables of domain size $d$ and the $S$ set is composed of the first $d / 2$ elements. We generate a family of instances by varying the size of $q$ and of the difference between min and $\max , \Delta=\max -\min$. For each family we try to generate 10 challenging instances by randomly filling the domain of each variable and by enumerating all possible values of min. These instances are then solved using a random choice for both variable and value selection, keeping only the ones that are solved with more than 10 backtracks by method IB. All runs were stopped after one minute of computation.

Table1 1reports on instances with a fixed number of variables (100) and varying $q$ and $\Delta$. Table 2 reports on instances with a fixed $\Delta(1)$ and growing number of variables. The results confirm that the new algorithms are very efficient. The average number of backtracks for SLG is generally very low. As predicted by its time complexity, GS is very stable for fixed $n$ in the first table but becomes more time consuming as $n$ grows in the second table. The performance of SLG and REG decreases as $q$ grows but REG remains competitive throughout these 
Table 3. Comparison on small car sequencing instances

\begin{tabular}{|r|rr|rr|}
\hline Version & \multicolumn{2}{|c|}{ Average } & \multicolumn{2}{c|}{ Median } \\
& BT & CPU & BT & CPU \\
\hline A & 1067 & 26.5 & 0 & 4.6 \\
B & 1067 & 10.3 & 0 & 3.8 \\
C & 802 & 8.4 & 0 & 4.1 \\
D & 798 & 34.3 & 0 & 7.0 \\
\hline
\end{tabular}

tests. We expect that the latter would suffer with still larger values of $q$ and $\Delta$ but it proved difficult to generate challenging instances in that range - they tended to be loose enough to be easy for every algorithm.

\subsection{Car Sequencing}

In order to evaluate this constraint in a more realistic setting, we turned to the car sequencing problem. We ran experiments using the first set of instances on the CSPLib web site and out of the 78 instances we kept the 31 that could be solved within 5 minutes using a program found in the Ilog distribution. Recall that the Ilog version of the sequence constraint also allows to specify individual cardinalities for values in $S$ so it is richer than our version of sequence . Table 3 compares the following versions of the sequencing constraint: (A) original Ilog program; (B) A + REG (added as a redundant constraint); (C) A + REG with cost [5, using the cost variable to restrict the total number of cars with a particular option; (D) A + REG with cost, using the cost variable to restrict the total number of cars of a particular configuration for each option. For A,B and $\mathrm{C}$ we thus introduce one constraint per option and for D we add one constraint per configuration and option.

It is interesting to see that adding REG as a redundant constraint significantly improves performance as it probably often detects a dead end before IloSequence does, thus avoiding expensive work. The simple cost version (C) does quite well since it also incorporates a weak form of cardinality constraint within the sequence constraint. For a fairer comparison, we chose not to compare our two other algorithms as we do not currently have incremental implementations.

\section{Discussion}

We have proposed, analyzed, and evaluated experimentally three filtering algorithms for the sequence constraint. They have different strengths that complement each other well. The local graph approach of Section 4 does not guarantee domain consistency but causes quite a bit of filtering, as witnessed in the experiments. Its asymptotic time complexity is $O\left(n q^{2}\right)$. The reformulation as a regular constraint, described in Section 15, establishes domain consistency but its asymptotic time and space complexity are exponential in $q$, namely $O\left(n 2^{q}\right)$. Nevertheless for small $q$, not uncommon in applications, it performs very well partly due to its incremental algorithm. The generalized sequence approach of 
Section[6]also establishes domain consistency on the sequence constraint, as well as on a more general variant defined on arbitrary among constraints. It has an asymptotic time complexity that is polynomial in both $n$ and $q$, namely $O\left(n^{3}\right)$. Also in practice this algorithm performed very well, being often even faster than the local graph approach. It should be noted that previously known algorithms did not establish domain consistency.

Since $q$ plays an important role in the efficiency of some of the approaches proposed, it is worth estimating it in some typical applications. For example, in car sequencing values between 2 and 5 are frequent, whereas the shift construction problem may feature widths of about 12.

As a possible extension of this work, our first two algorithms lend themselves to a generalization of sequence in which the number of occurrences is represented by a set (as opposed to an interval of values).

\section{Acknowledgments}

We thank Marc Brisson and Sylvain Mouret for their help with some of the implementations and experiments, as well as the referees for their comments.

\section{References}

1. K.R. Apt. Principles of Constraint Programming. Cambridge Univ. Press, 2003.

2. N. Beldiceanu and M. Carlsson. Revisiting the Cardinality Operator and Introducing the Cardinality-Path Constraint Family. In ICLP 2001, volume 2237 of LNCS, pages 59-73. Springer, 2001.

3. N. Beldiceanu, M. Carlsson, and J.-X. Rampon. Global Constraint Catalog. Technical Report T2005-08, SICS, 2005.

4. N. Beldiceanu and E. Contejean. Introducing global constraints in CHIP. Journal of Mathematical and Computer Modelling, 20(12):97-123, 1994.

5. S. Demassey, G. Pesant, and L.-M. Rousseau. A cost-regular based hybrid column generation approach. Constraints. under final review.

6. R. Mohr and G. Masini. Good Old Discrete Relaxation. In European Conference on Artificial Intelligence (ECAI), pages 651-656, 1988.

7. G. Pesant. A Regular Language Membership Constraint for Finite Sequences of Variables. In CP 2004, volume 3258 of $L N C S$, pages 482-495. Springer, 2004.

8. J.-C. Régin. Generalized Arc Consistency for Global Cardinality Constraint. In AAAI/IAAI, pages 209-215. AAAI Press/The MIT Press, 1996.

9. J.-C. Régin. Combination of Among and Cardinality Constraints. In CPAIOR 2005, volume 3524 of $L N C S$, pages 288-303. Springer, 2005.

10. J.-C. Régin and J.-F. Puget. A Filtering Algorithm for Global Sequencing Constraints. In $C P^{\prime} 97$, volume 1330 of $L N C S$, pages 32-46. Springer, 1997.

11. M.A. Trick. A Dynamic Programming Approach for Consistency and Propagation for Knapsack Constraints. Annals of Operations Research, 118:73-84, 2003. 